SUB-BARRIER REACTIONS MEASURED USING A

RECOIL MASS SEPARATOR

CONF-8806167--6

DE89 005803

R. R. Betts

Physics Division, Argonne National Laboratory

Argonne, IL 60439, U.S.A.

\author{
Invited Paper \\ presented at \\ Symposium on \\ HEAVY ION INTERACTIONS AROUND THE \\ COULOMB BARRIER
}

Legnaro, Italy 1-4 June 88

To be published by Springer-Verlag in

"Lecture Notes in Physics"
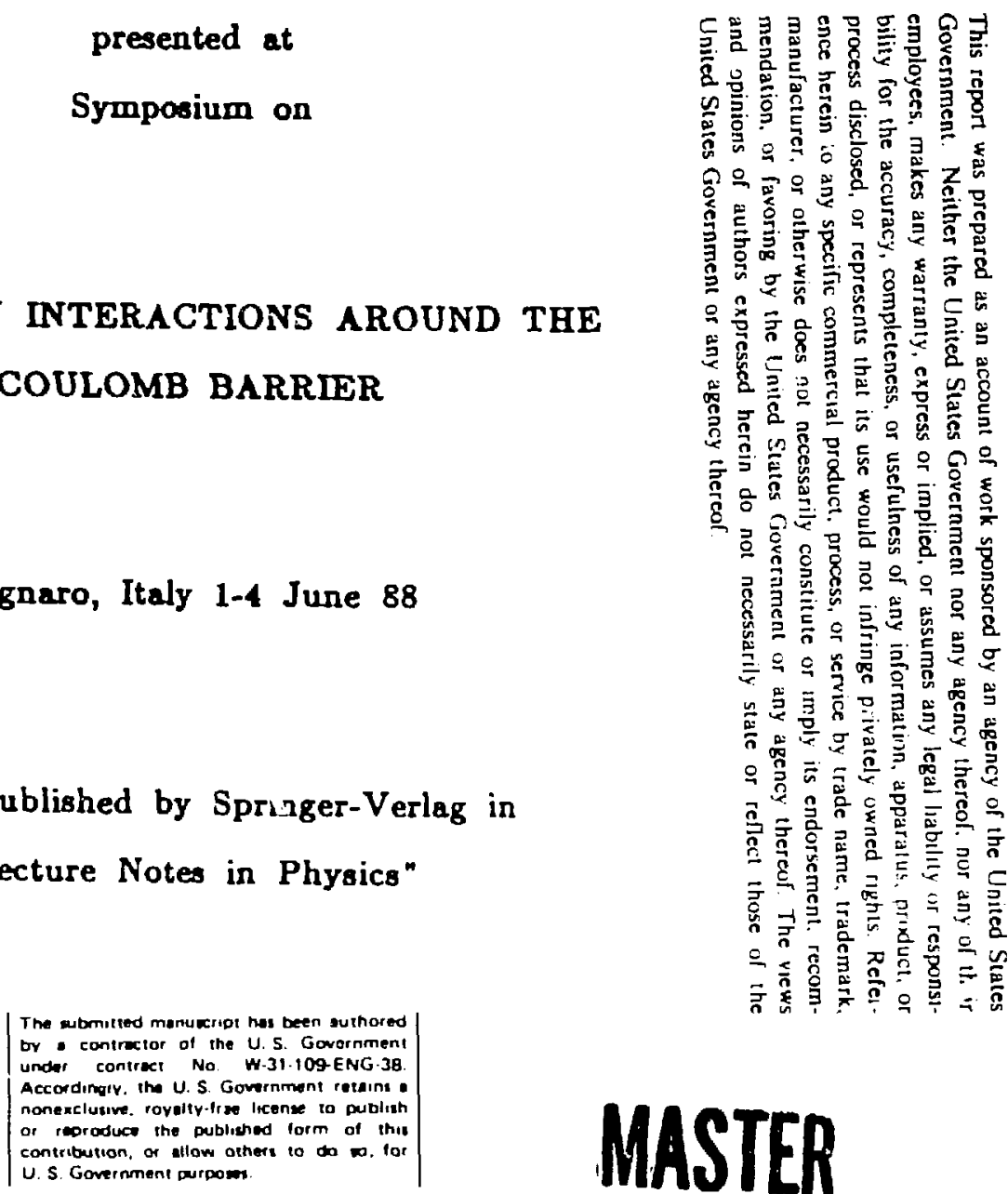


\title{
SUB-BARRIER REACTIONS MEASURED USING A \\ RECOIL MASS SEPARATOR
}

\author{
R. R. Betts \\ Physics Division, Argonne National Laboratory \\ Argonne, IL 60439, U.S.A.
}

Much recent effort [1] has been directed to the study of the interaction of heavy nuclei at energies close to and below their mutual classical interaction barrier. This incerest is motivated by the observation of characteristics of nuclear reactions at these energies which cannot be understood in terms of simple first order models. The most graphic example of this being, of course, the observed enhancement of the cross-section for fusion of heavy ions at sub-barrier energies. Our current understanding of this phenomenon involves the influence of the coupling between elaztic, quasi-elastic and fusion channels which leads to an interplay of nuclear structure and dynamice resulting in the observed enhancements to the fusion channel. In principle, therefore, a framework exists for a self-consistent description of all features of nuclear interactions in the sub-barrier region. In addition, the understanding of "ransfer between heavy ions is important in its own right. The hope being that new nuclear structure information might be obtained from such reactions. The sub-barrier region, in which the number of competing reaction channels is minimal, should therefore be the first testing ground of this understanding.

Despite these strong motivations, few data exist in the sub-barrier region for reaction channels other than fusion. In particular, our experimental knowledge of quasi-elastic transfer reactions is sparse, despite the belief that this particular channel may be dominant in determining some features of the sub-barrier fusion enhancement. This deficiency is due in part to experimental difficulties. Transfer reactions are governed primarily by the closest approach of the colliding nuclei which, at low energies, results in a strong backward peaking of the angular distribution in the center-of-mass frame. For situations where the projectile has a significant fraction of the target mass, as is so in most cases of interest, the backscattered projectile-like fragment has such low energy that the usual techniques of measurement and identification become invalid. Here, we report on a solution to this problem which allows a systematic study of many aspects of transfer reactions in the energy regime of interest. We exploit the fact that associated with the lowenergy backscattered projectile-like fragment is complementary target-like fragment which recoils to forward angles with a large fraction of the incident beam energy. These target-like fragments were detected and identified using the Daresbury Recoil 
Mass Separator (RMS) thus allowing the measurement of quasi-elastic trangfer over a hitherto inaccessible energy range from the vicinity of the barrier to several tens of $\mathrm{MeV}$ below.

The Daresbury R.MS has been described in the literature [2] and recent developments are discussed elsewhere in these proceedings. [3] Briefly, it consists of a velocity filter followed by momentum selection giving an image in the focal plane dispersed in A/q. The position of the transmitted ions in the focal plane is measured using a position sensitive channel plate detector and their specific esergy loss and total energy are then measured using an ionization chamber. The experiments described here used ${ }^{58} \mathrm{Ni}$ beams of energies ranging from 180 to 260 $\mathrm{MeV}$ provided by the Daresbury Laboratory Nuclear Structure Facility tandem accelerator. Data on sub-barrier transfer for cargets of $116,118,120,122,124 \mathrm{Sn}[4,5]$ and $114,148,150,152,154$ Sm were obtained.

A spectrum of energy loss versus total energy for recoils detected in the bombardment of ${ }^{122} \mathrm{Sn}$ with a $214 \mathrm{MeV}$ beam of ${ }^{58} \mathrm{Ni}$ is shown in Fig. 1. The $\mathrm{Sn}$ -

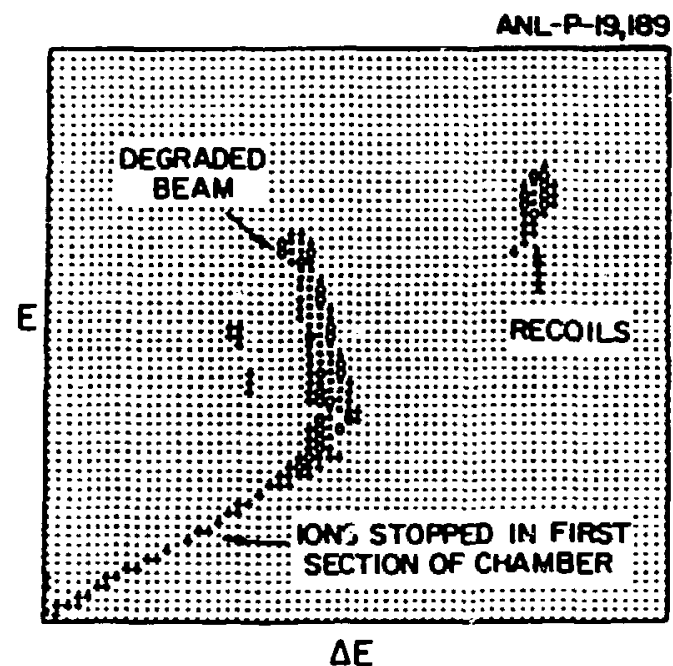

Fig. 1. Two-dimensionai spectrum of energy loss versus total energy for recoils from $214 \mathrm{MeV}^{58} \mathrm{Ni}$ $+{ }^{122} \mathrm{Sn}$.

like recoils are identified by their large energy loss compared to a tail of degraded beam particles produced by scattering from the electrostatic plates in the velocity 
filter. The distribution of the Sn-like events as anction of energy and focal-plane position is shown in Fig. 2 together with the projections

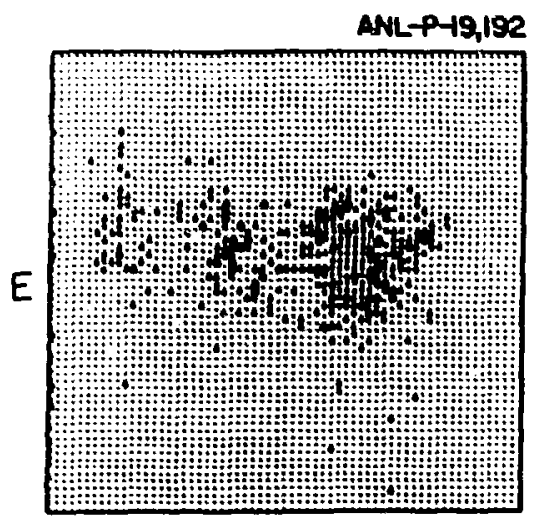

Alq
Fig. 2. Two-dimensional spectrum of position versus total energy for Sn-like events. The projections on to the position and energy axes are also shown.

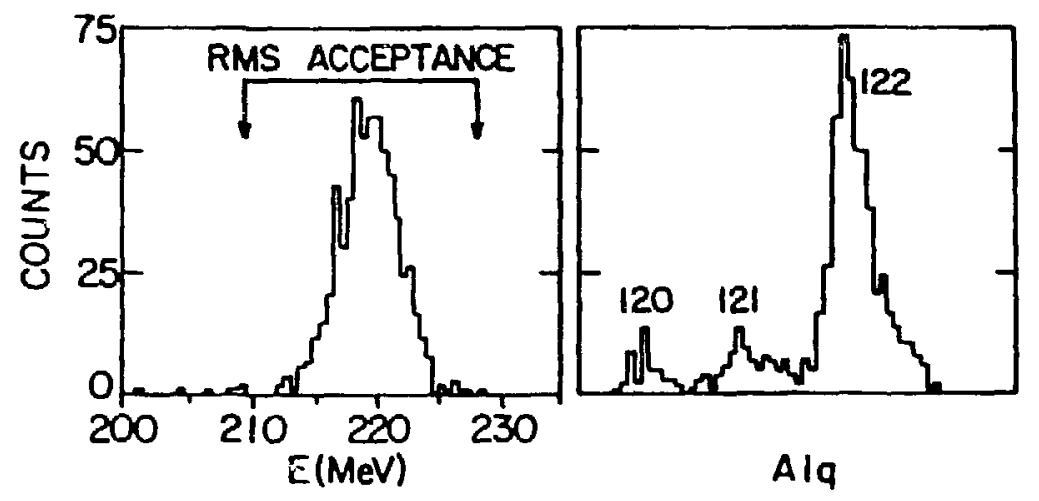

of these events onto the two axes. The better than unit mass resolution of the RMS is evident from the position spectrum which, in this case, shows peaks corresponding to masses 122,121 and 120 all of charge-state $28^{+}$. The energy spectrum shows a broad peak which, nevertheless, falls well within the acceptance of the recoil separator as indicated on the spectrum. This observation is further confirmed by a scan of the yield as a function of the central velocity setting of the Wien filter which gives a distribution of width equal to the expected velocity acceptance $(\Delta v / v \simeq 4 \%)$ consistent with the velocity spread of the detected fragments being narrower than the RMS acceptance.

On the basis of Q-values and the expected fall off of form factors at large radius, as well as the results of DWBA calculations, we expect that the observed yields correspond to neutron, rather than proton, transfer. This expectation is confirmed by the results of a measurement of coincident gamma rays in the case of 
${ }^{58} \mathrm{Ni}+{ }^{124} \mathrm{Sn}$ at a bombarding energy of $220 \mathrm{MeV}$. In this case the one nucleon transfer channel is conclusively identified as neutron transfer. These results are discussed in more detail in another paper [6].

Cross sections were obtained by assuming that the total yield observed corresponds to the Rutherford scattering cross-section; the crose-sections for individual masses then being given by the ratio of their yields to the total. The validity of this procedure was verified in cases where the yields were measured for all the strong charge states and a cross-section calculated using the ratios of the solid angles of the RMS and a monitor detector fixed at $36.5^{\circ}$ together with the measured yield in the monitor counter. The cross-sections obtained by the two methods agreed to within $15 \%$.

Cross sections for ${ }^{58} \mathrm{Ni}+{ }^{116,118,120,122,124} \mathrm{Sn}$ one-neutron pickup reactions are shown plotted as a function of center-of-mass bombarding energy in Fig. 3. The data for each isotope fall with decreasing bombarding energy with

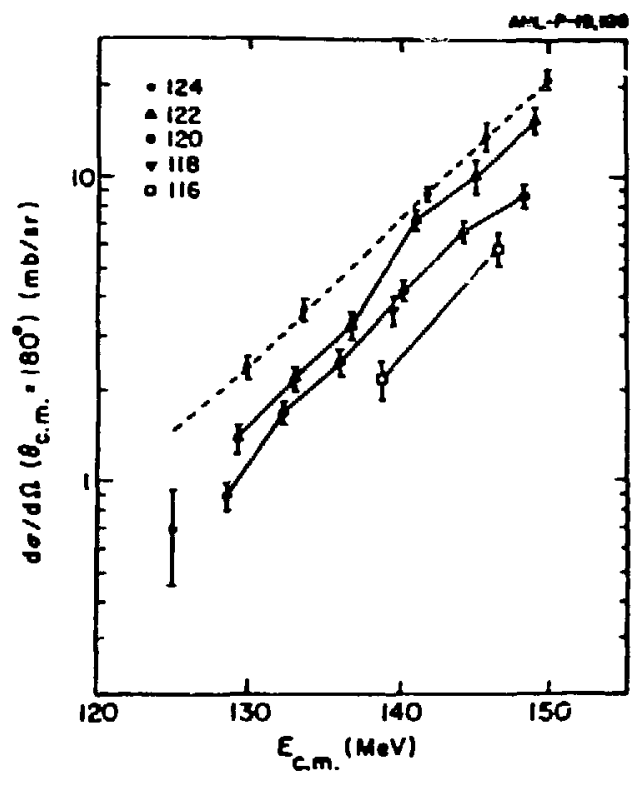

Fig. 3. Cross-sectigns for one-nucleon pickup in ${ }^{58} \mathrm{Ni}+\mathrm{Sn}_{\mathrm{n}}$ reactions plotted versus center-of mass bombarding energy. The dashed curve shows the DWBA prediction for ${ }^{24} \mathrm{Sn}$. The solid lines join the data points.

slopes which sre independent of the target. The absolute magnitudes, however, depend strongly on target mass, varying by a factor of three from the heaviest to the lightest isotope. The general features of these data can be understood ss a result of the monotonic change in the ground-state neutron binding energy across the isotopic sequence. This, coupled with the almost unchanging collective and single-particle structure of the $\mathrm{Sn}$ isotopes results in the rather simple behavior observed. Table I lists some of the relevant properties of the $\mathrm{Sn}$ isotopes studied. 


\section{Table I}

Properties of the Eve, Sn Isotopes

$\begin{array}{cccccc} & B_{n} & Q_{n} & \pi_{n} & & \\ f^{-1} & \mathrm{MeV} & \mathrm{MeV} & \mathrm{fm}^{\mathrm{a}} & \rho_{3}{ }^{\mathrm{a})}\end{array}$

$\begin{array}{llrlll}116 & 9.57 & -0.56 & 0.681 & 0.120 & 0.046 \\ 118 & 9.33 & -0.33 & 0.672 & 0.110 & 0.035 \\ 120 & 9.10 & -0.11 & 0.664 & 0.108 & 0.031 \\ 122 & 8.80 & 0.18 & 0.653 & 0.104 & 0.035 \\ 124 & 8.49 & 0.51 & 0.641 & 0.095 & 0.041\end{array}$

a) Deformation associated with the collective inelastic excitation of the lowest $2^{+}$and $3^{-}$states

Within the framework of a simple semi-classical treatment of neutron transfer, we expect the dependence of the $\theta_{\mathrm{cm}}=180^{\circ}$ cross-section to be given by;

$$
\frac{d \sigma}{d a} \times\left.\frac{e^{-2 x_{n} d}}{x_{n}} \frac{d \sigma}{d \pi}\right|_{\text {Rutherford }} \text { where } d=\frac{z_{1} z_{2} e^{2}}{E_{c m}},
$$

the distance of closest approach for a head-on collision. The cross-sections calculated with this expression are shown in Fig. 4 together with the data. The

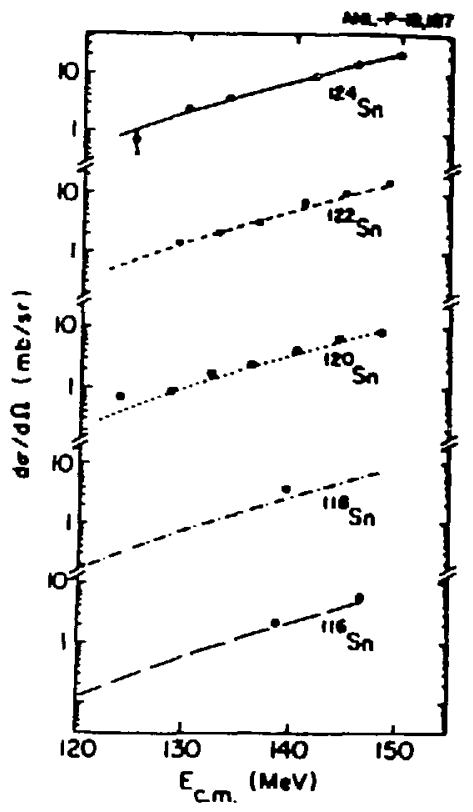

Fig. 4. Gross-sections for one-nucleon pickup in ${ }^{58} \mathrm{Ni}+\mathrm{Sn}$ together with cross-sections calculated using the semi-classical expression given in the text. The curves are normalized to the highest energy point for ${ }^{12} \mathrm{Sn}$.

calculated curves were normalized to the data at one point, that for ${ }^{124} \mathrm{Sn}$ at the highest energy. The overall agreement is quite remarkable. The near constancy of 
the observed slopes can be seen from the form of the semi-classical expression which has a logarithmic slope which depends only linearly on $x_{n}$ - and remains constant within a few percent scross the isotopic sequerce. The magnitude of the crosssection is, however, exponentially dependent on the product of $x_{n}$ and $d$ which leads to the observed strong dependence. It would not have been expected a priori that this simple treatment give such a good account of the variation of the absolute magnitudes of the cross-sections. This has the additional requirement that the dependence of the overall magnitude of the valence neutron wave function at the nuclear surface vary only weakly from isotope to isotope such that its magnitude at large distances is governed solely by $x_{n}$ the binding energy parameter.

A more detailed analysis of these data has been carried out in DWBA using spectroscopic information taken from light ion studies $[7,8]$ in this mass region together with the code PTOLEMY. [9] The resultant energy dependence is in similarly good agreement with the data, as is the isotopic dependence which is shown together with the data and the semi-classica! results in Fig. 5. The

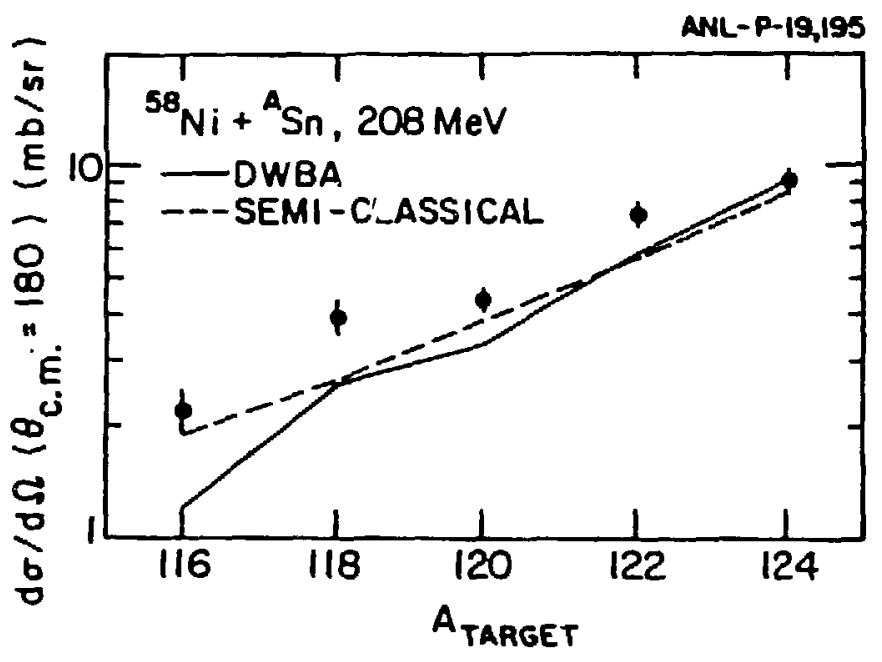

Fig. 5. Cross-sections for onepucleon pickup in ${ }^{58} \mathrm{Ni}+\mathrm{Sn}$ at 208 $\mathrm{MeV}$ together with the predictions of the semi-classical model and the results of DWBA calculations.

conclusion is therefore that the sub-barrier neuwron transfer of ${ }^{58} \mathrm{Ni}+\mathrm{Sn}$ is well understood within the framework of simple semi-classical and quantum mechanical theories of the transfer process.

The influence of the transfer process on the sub-barrier fusion of these systems has been investigated using the model of Dasso et al., [10] which provides a transparent connection between the strengths of the quasi-elastic reaction channels 
and fusion. The results of these calculations are shown in Fig. 6 which shows, successively from left to right, the cruss-sections

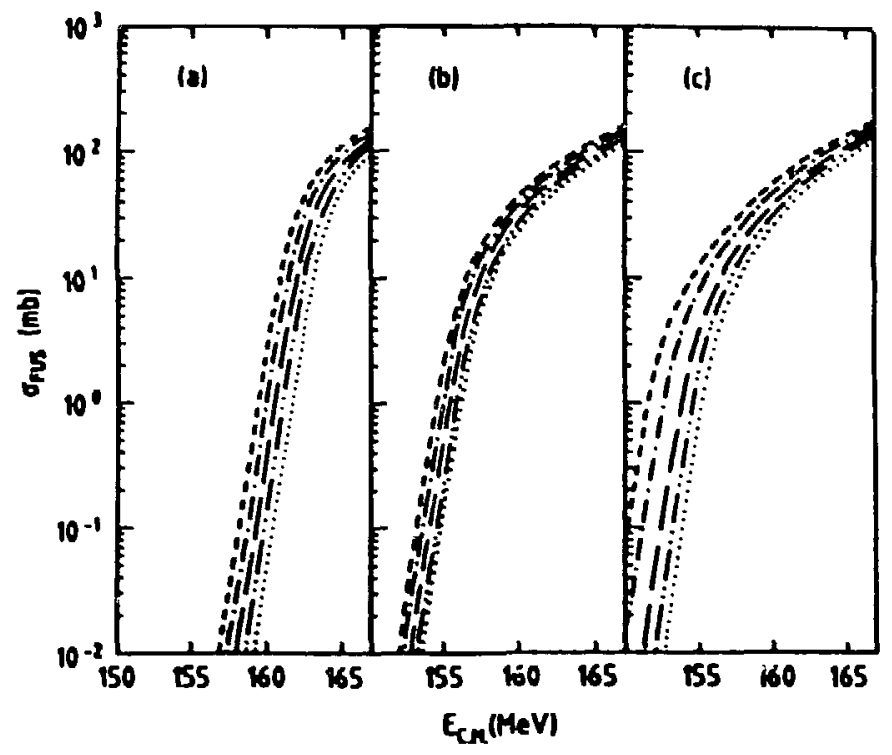

Fig. 6. Calculated fusion cross-sections for $\mathrm{Ni}$ + Sn. Figures a) $-c$ ) show the results using the bare potential, bare + inelastic and bare + inelastic + transfer. Figures d)-f) show the same resuits corrected for geometric effects as discussed in the text.

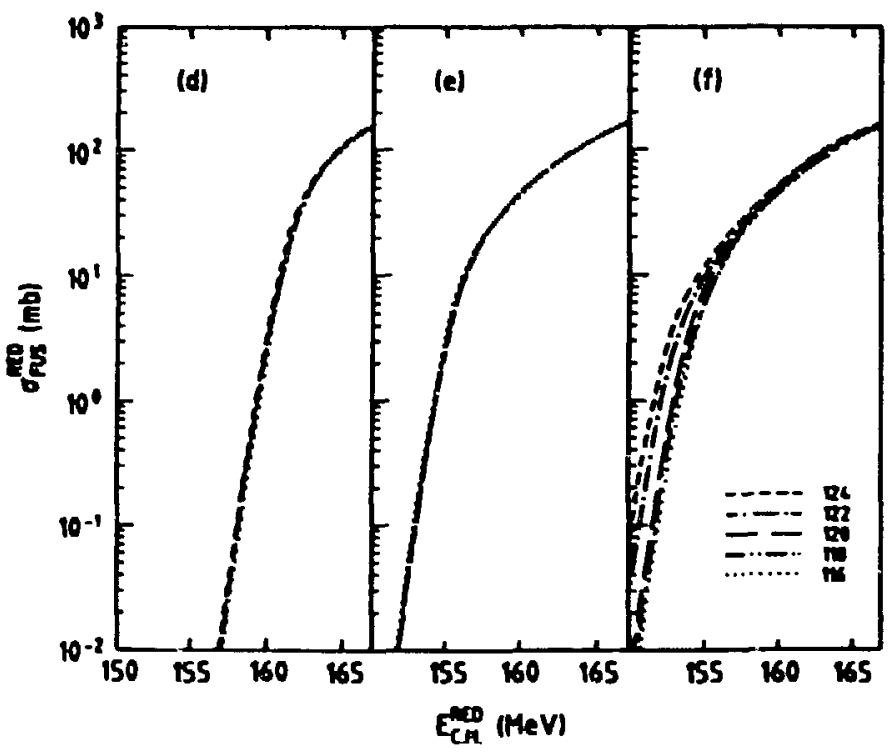

calculated with no-coupling, inelastic coupling and, inelastic + transfer coupling.

The relative strengths of the transfer coupling ware taisen from the present measured cross-sections. The lower portion of the figure shows the effect of removing geometric effects on the isotopic dependence through defining the appropriately scaled quantities

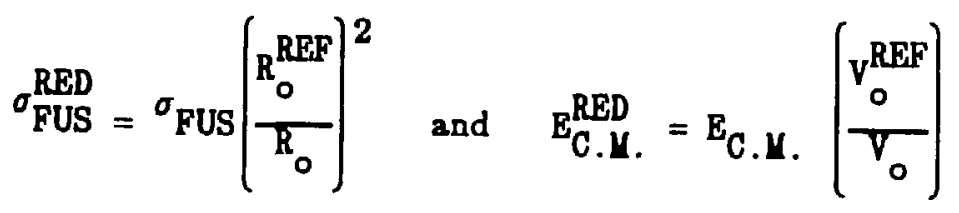


where $R_{0}$ and $V_{0}$ are the s-wave barrier heights and radii respectively. It is evident from these curves, that the only non-trivial isotopic dependence of the sub-barrier fusion of these systems resuits from the coupling of the fusion channel to the otrongly varying one-neutron transfer channel. These results are in fair agreement with the available data [11] for low-energy fusion of these systems although a more detailed experimental study of the sub-barrier fusion is now clearly merited.

The results for ${ }^{58} \mathrm{Ni}+\mathrm{Sm}$ are less easily understood. Table II lists some of the properties of the isotopes stucied which, in ccritrast to $\mathrm{Sn}$, show a transition from spherical $\left({ }^{144} \mathrm{Sm}\right)$ to well-deformed $\left({ }^{154} \mathrm{Sm}\right)$. The cross-sections for one-neutron pickup are shown plotted versus center-of-mass bombarding energy in Fig. 7 . As in the case of $\mathrm{Sn}$ there is a strcng isotopic dependence of the absolute magnitudes but in

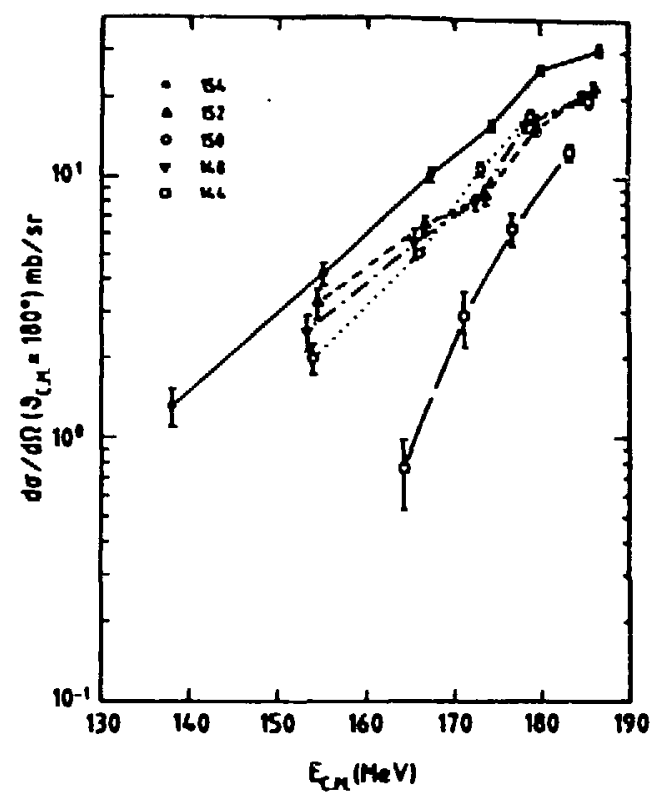

Fig. 7. Cross-sections for one-nucleon pickup in $\mathrm{Ni}+\mathrm{Sm}$ reactions plotted versus center-of-mass bombarding energy.

Table II

Properties of the Even Sm Isotopes

$\begin{array}{crrrr} & \begin{array}{c}B_{n} \\ \Lambda\end{array} & \begin{array}{c}Q_{n} \\ M e V\end{array} & \begin{array}{c}\kappa_{n} \\ f_{m}^{-1}\end{array} & \left.\rho_{2}{ }^{2}\right) \\ 144 & 10.55 & -1.56 & 0.715 & 0 . \\ 148 & 8.14 & 0.86 & 0.828 & 0.11 \\ 150 & 7.99 & 1.01 & 0.622 & 0.19 \\ 152 & 8.27 & 0.73 & 0.633 & 0.25 \\ 154 & 7.98 & 1.02 & 0.622 & 0.27\end{array}$

a) Static ground-state deformation.

addition there now appears a dramatic variation in slope between the spherical ${ }^{144} \mathrm{Sm}$ target and thi eformed targets. This variation in slope is in excess of that 
expected on the basis of the change of $2.5 \mathrm{MeV}$ in binding energy between ${ }^{144} \mathrm{Sm}$ and the deformed targets as is illustrated in Fig. 8 where the data are

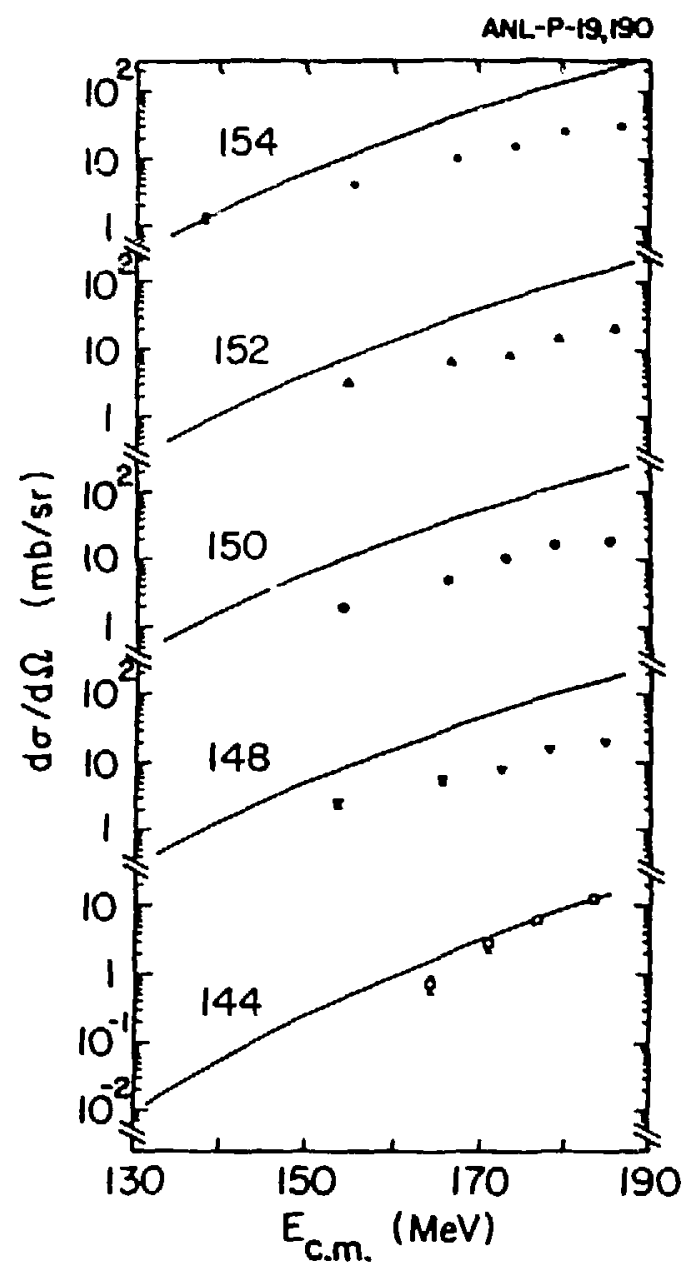

Fig. 8. Comparison between measured cross-sections for ${ }^{{ }^{8}} \mathrm{Ni}+\mathrm{Sm}$ transfer and cross-sections calculated using the semiclassical model. The calculations are normalized to the highest energy point for $\mathrm{Sm}$.

compared to the predictions of the semi-classical model. For ${ }^{144} \mathrm{Sm}$ the calculation is in good agreement with the observed slope but for the heavier targets the observed slopes are much less than the semi-classical prediction. In fact, to reproduce the observed slope for ${ }^{154} \mathrm{Sm}$, an effective binding energy of $3.6 \mathrm{MeV}$ is required - some $4.4 \mathrm{MeV}$ less than the ground-state value. $A$ similar behavior is 
observed in the comparison between the data ard the results of DWBA calculations shown in Fig. 9. A.s for $\mathrm{Sn}$, the spectroscopic information

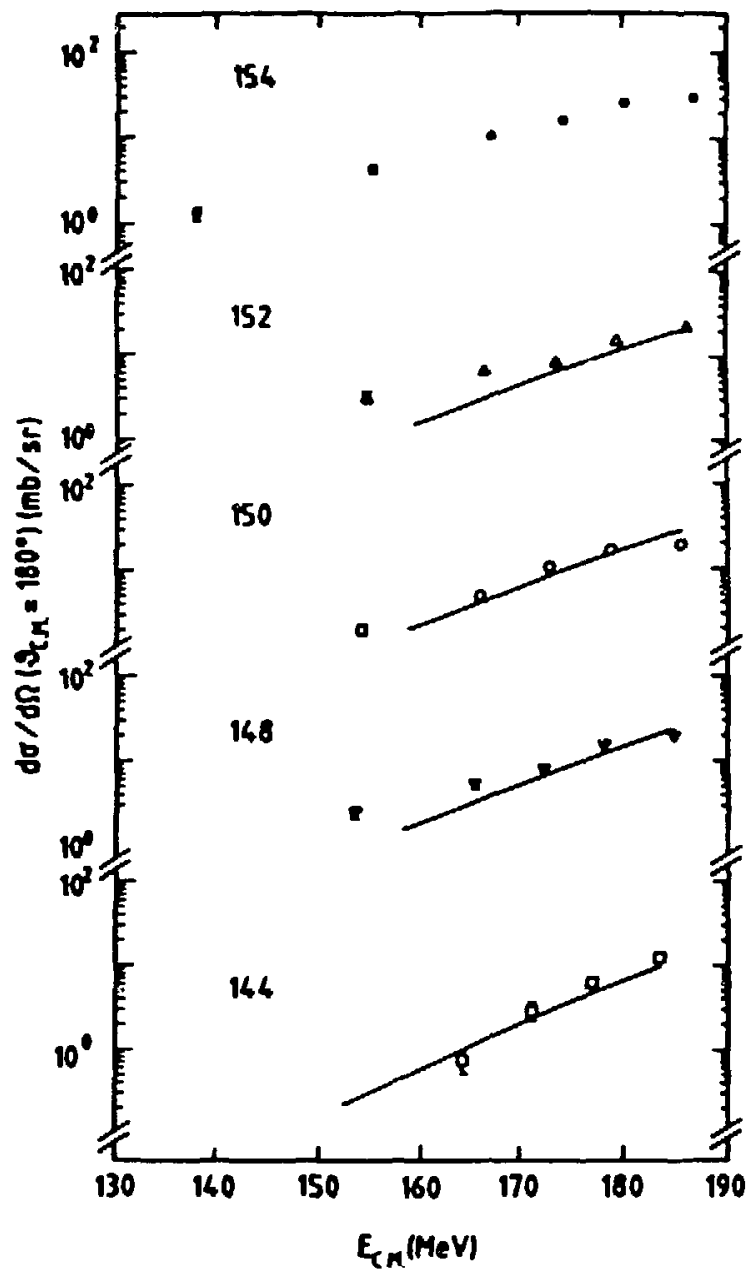

Fig. 9. Comparison between measured cross-sections for ${ }^{58} \mathrm{Ni}+\mathrm{Sm}$ and the results of DWBA calculations.

was taken from light ion work. [12] The DWEA predictions are in good agreement with the observed magnitudes at the highest energies but fail to predict the fall in cross-section with decreasing energy for the deformed targets, the calculated crosssections falling too steeply. We note that the ${ }^{144} \mathrm{Sm}$ data are well accounted for over the entire energy range studied.

Within the framework of the semi-classicai model, it might be suspected that the observed changes in slope result simply from the additional quadrupole term in the Coulomb potential which arises from the deformed target charge distributions. This effect is readily included in the semi-classical formalism $[13,14]$, but for randomly oriented target nuclei leads to an effect which is both small and in the wrong direction, the calculated curves now becoming slightly steeper than for a spherical target. The assumption of prolate target nuclei all aligned with their symmetry axis along the beam direction does produce a decrease in the slope over 
the spherical case but, for reasonable deformations, is still somewhat smaller than that observed. [14]

It is interesting to note the similarity of the present observations to those of Wirth et al., for ${ }^{238} U+{ }^{258} U$ at sub-barrier energies. [15] The present data for ${ }^{154} \mathrm{Sm}$ are compared with the $\theta_{\mathrm{cm}}=180^{\circ}$ data points for ${ }^{238} \mathrm{U}+{ }^{238} \mathrm{U}$ in Fig. 10. The two lines are, in each case, a line drawn through the data

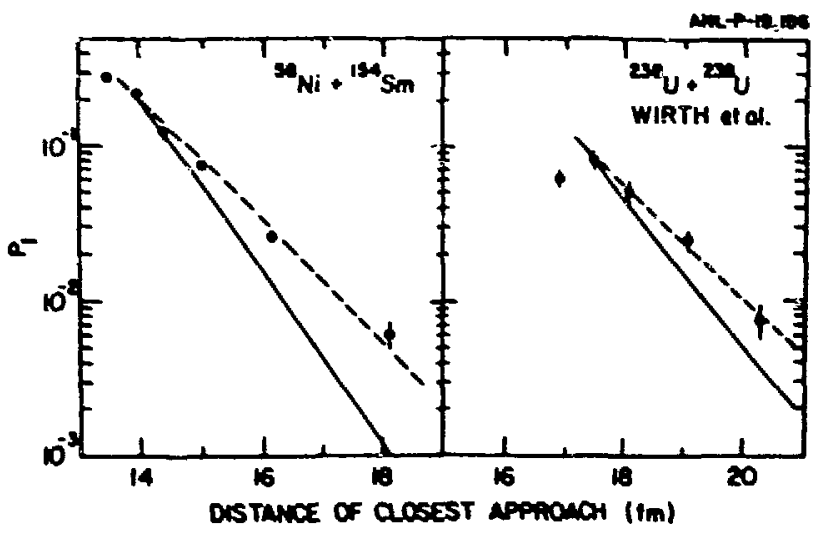

Fig. 10. Comparison betwean transfer Probabilities for ${ }_{+}^{58}{ }^{58}+{ }_{U}^{+}{ }^{5 m}$ and the predictions of the semiclassical model using ground-state binding energies (orilid curve). The dashed curve through the points is to guide the eye.

to guide the eye and the semi-classical expectation using ground state binding energies. In both cases the enhancement of the data over the semi-classical expectation is similar. The ${ }^{258} U+{ }^{258} U$ data are of particular interest in that it has been found for angles corresponding to more glancing collisions the data were well accounted for by the semi-classical calculation which indicates that the enhancement of transfer is connected to head-on rather than larger impact parameter collisions. It appears therefore that the enhancement of transfer at large distances is a quite general effect associated with the interactions between heavy ions in which at least one of the reaction partners is deformed. It does not seem that the magnitude of the deformation has any significant effect, as the enhancement appears similar between the more weakly deformed and strongly deformed Sm isotopes and between all of these and the well deformed-deformed ${ }^{258} U+{ }^{228} U$ case.

Similar "slope anomalies" have been reported by Juutinen et al., [16] for several different projectiles incident on Dy target nuclei all at bombarding energies above the barrier. In these cases, in which gamma ray energy and multiplicity information were als obtained, it was concluded that the occurrence of the slope anomaly was correlated with small angular momentum transfer. In the present data it is true that the low bombarding energy and detection angle also favor low angular momentum transfer but this evidence is only circumstantial. It seems 
nevertheless that such anomalies maybe a general phenomenon and that further study will give us new insight into the interplay between structure and dynamics in heavy ion reactions.

This work was perfurmed in collaboration with C.N. Pass, F.M. Evans, A.E. Smith (Oxford); J.S. Lilley, J. Simpson, J.R. Smith (Daresbury); A.N. James (Liverpos'); and B.R. Fulton (Birmingham). Support from NATO Collaborative Research Grant $0076 / 87$ is gratefully acknowledged. This research was supported by the U.S. Department of Energy, Nuclear Physics Division, under contract

W-31-109-ENG-38.

\section{References}

(1) Proceedings of the International Conference on Fusion Reactions below the Coulomb Rarrier, Cambridge, Mass. June 1984 ed. S.G. Steadman (Springer Verlag, Berlin 1985).

[2] A.N. James, et al., Nucl. Inst. and Meth. A267, 144 (1988).

[3] A.N. James, these proceedings.

[4] R.R. Betts, et 으., Phys. Rev. Lett. 59,978 (1987).

[5] C.N. Pass et al., submitted to Nucl. Phys.

[6] C.N. Pass et al; these proceedings.

[7] E.J. Schueid et aㅡ, Phys. Rev. 156, 1316 (1967).

[8] P.E. Cavanagh et al., AERE Harwell Report No. AERE-R5901 (unpublished).

[9] S.C. Pieper et al., ANL Report No. ANL-76-11 (unpublished).

[10] C.H. Dasso, S. Landowne and A. Winther, Nucl. Phys. A407, 221 (1983).

[11] K.T. Lesko et al., Phys. Rev. C34, 2155 (1988j.

[12] S. Gales al; Nucl. Phys. A398, 19 (1983).

[13] R.K. Gupta et al., Phys. Rev. C37, 1502 (1988).

[14] R.R. Betts et al., to be published.

[15] G. Wirth et al., Phys. Lett. B177, 282 (1986).

[16] S. Juutinen et a․, Phys. Lett. B192, 307 (1987). 